RESEARCH ARTICLE

\author{
Dario Fasino • Domenico Freni
}

\title{
Fundamental relations in simple and 0-simple semihypergroups of small size
}

Received: 3 November 2011 / Accepted: 8 February 2012 / Published online: 5 April 2012

(C) The Author(s) 2012. This article is published with open access at Springerlink.com

\begin{abstract}
We consider the fundamental relations $\beta$ and $\gamma$ in simple and 0-simple semihypergroups, especially in connection with certain minimal cardinality questions. In particular, we enumerate and exhibit all simple and 0 -simple semihypergroups having order 3 where $\beta$ is not transitive, apart of isomorphisms. Moreover, we show that the least order for which there exists a strongly simple semihypergroup where $\beta$ is not transitive is 4. Finally, we prove that $\gamma$ is transitive in all simple semihypergroups, and determine necessary and sufficient conditions for a 0 -simple semihypergroup to have $\gamma$ transitive. The latter results obviously hold also for simple and 0 -simple semigroups.
\end{abstract}

Mathematics Subject Classification (2010) 20N20 - 05A99

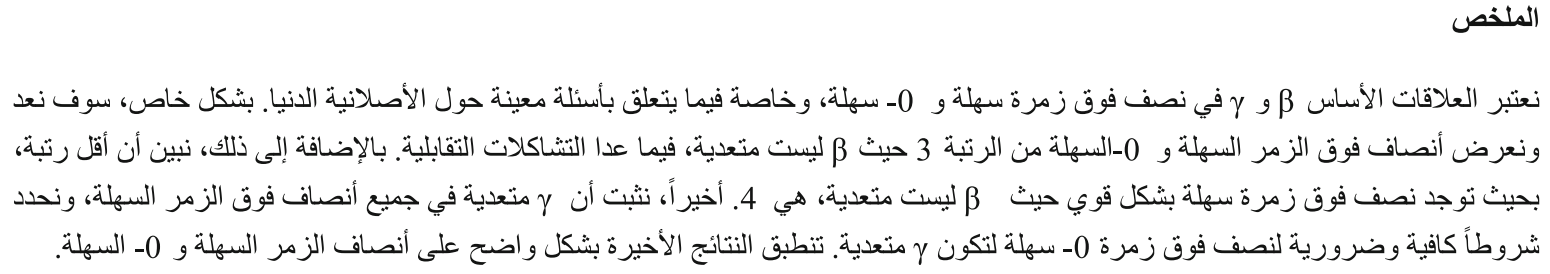

\section{Introduction and preliminaries}

Among algebraic hyperstructures, semihypergroups are currently attracting a growing interest being subject of study by various authors, and under different perspectives $[2,3,8,9,13-15,18]$. For example, interesting problems arise in the study of their so-called fundamental relations, which lead to analyse conditions for their transitivity $[11,12,16]$, algebraic properties of the associated quotient structures $[3,4,18,21]$, and minimal cardinality problems $[8,9,13]$. In particular, in the paper [13] it is shown that in all semihypergroups $H$ of type

D. Fasino $(\varangle)$

Dipartimento di Chimica, Fisica e Ambiente, Università di Udine,

Udine, Italy

E-mail: dario.fasino@uniud.it

D. Freni

Dipartimento di Matematica e Informatica, Università di Udine,

Udine, Italy

E-mail: domenico.freni@uniud.it 
$U$ on the right the fundamental relation $\beta$ is transitive if $|H| \leq 8$, and exhibits a counterexample having order 9; furthermore, in all semihypergroups of type $U$ on the right the fundamental relation $\gamma$ is always transitive. The above mentioned results allowed to solve various minimal cardinality problems on finite semihypergroups of type $U$ on the right.

The present paper deepens the knowledge of the fundamental relations $\beta$ and $\gamma$ in the classes of simple and 0 -simple semihypergroups, and addresses certain minimal cardinality problems in connection with them. Simple and 0-simple semihypergroups have been recently introduced in [18] as a generalization of analogous well known and widely studied structures in semigroup theory [17]. We remark that the class of simple semihypergroups extends that of finite semihypergroups of type $U$ on the right. Motivated by the results in [13], it is natural to consider whether or not the relations $\beta$ and $\gamma$ are transitive in the wider class of simple semihypergroups. Actually, we prove hereafter that $\gamma$ is transitive in all simple semihypergroups, while the least order of a simple semihypergroup having $\beta$ non transitive is 3 . Analogous properties are also found in the class of 0 -simple semihypergroups.

The outline of this paper is as follows: After introducing some fundamental definitions and results, in Sect. 2 we recall definitions and elementary properties of simple and 0 -simple semihypergroups, and we define strongly simple semihypergroups, a subclass of simple semihypergroups which plays an important role in what follows. Furthermore, we give a list of examples and some special constructions of simple and strongly simple semihypergroups. Some of these examples will be exploited in successive sections. In Sect. 3 we determine all simple semihypergroups having order 3, apart of isomorphisms, where $\beta$ is not transitive. Remarkably, all these semihypergroups are not strongly simple, since they are either left- or right-reproducible.

This fact motivates the study carried out in Sect. 4 where we prove the existence of strongly simple semihypergroups having order 4 where $\beta$ is not transitive. The proof is constructive and exploits a special one-element extension of the semihypergroups found in the preceding section. In Sect. 5 we consider 0 -simple semihypergroups, as their analysis cannot be carried out as in the simple case, and we determine all 0 -simple semihypergroups having order 3 where $\beta$ is not transitive. In Sect. 6 we consider the relation $\gamma$ in both simple and 0 -simple semihypergroups. In particular we prove that $\gamma$ is transitive in all simple semihypergroups, thus extending the analogous result concerning semihypergroups of type $U$ on the right [13]. Moreover, we show a necessary and sufficient condition for a 0 -simple semihypergroup to have $\gamma$ transitive. In the last section we draw some conclusions and state an open problem.

Remark 1.1 Throughout the paper, we will often show hyperproduct tables of semihypergroups. These tables are usually obtained after long arguments that are aimed at proving the existence of semihypergroups having certain properties. We inform the reader that, after these tables are obtained, we always check their associativity either by hand or by means of computer routines, as those described in [7]. Hence, the corresponding semihypergroups are correctly defined, even if this is not always explicitly stated in what follows.

\section{Basic definitions and results}

Throughout this paper we use just a few basic concepts and definitions that belongs to common terminology in hyperstructure theory. A semihypergroup is a set endowed with an associative hyperproduct. A semihypergroup $H$ is left-reproducible (respectively, right-reproducible) if $H x=H$ (respectively, $x H=H$ ) for all $x \in H$. A hypergroup is a semihypergroup that is both left- and right-reproducible, hence $x H=H x=H$, for all $x \in H$.

A non-empty subset $S$ of a semihypergroup $H$ is called a subsemihypergroup of $H$ if it is closed with respect to multiplication, that is, if $x y \subset S$ for all $x, y \in S$. If the subsemihypergroup $S$ is a semigroup, we say that $S$ is a subsemigroup of $H$.

Given a semihypergroup $H$, the relation $\beta^{*}$ of $H$ is the transitive closure of the relation $\beta=\cup_{n \geq 1} \beta_{n}$, where $\beta_{1}$ is the diagonal relation in $H$ and, for every integer $n>1, \beta_{n}$ is defined as follows:

$$
x \beta_{n} y \Longleftrightarrow \exists\left(z_{1}, \ldots, z_{n}\right) \in H^{n}:\{x, y\} \subset z_{1} \cdots z_{n} .
$$

Moreover, if $S_{n}$ denotes the symmetric group on $\{1,2, \ldots, n\}$, the relation $\gamma^{*}$ is the transitive closure of the relation $\gamma=\cup_{n \geq 1} \gamma_{n}$, where $\gamma_{1}=\beta_{1}$ is the diagonal relation and, for every integer $n>1, \gamma_{n}$ is defined as follows:

$$
x \gamma_{n} y \Longleftrightarrow \exists\left(z_{1}, \ldots, z_{n}\right) \in H^{n}, \quad \exists \sigma \in S_{n}: x \in z_{1} \cdots z_{n}, y \in z_{\sigma(1)} \cdots z_{\sigma(n)} .
$$


The relations $\beta, \beta^{*}, \gamma$ and $\gamma^{*}$ are called fundamental relations on $H$ [21]. Their relevance in semihypergroup theory stems from the following facts [11,19]: If $H$ is a semihypergroup (resp., a hypergroup), then the relation $\beta^{*}$ is the smallest strongly regular equivalence on $H$ and the quotient $H / \beta^{*}$ is a semigroup (resp., a group). Moreover, the relation $\gamma^{*}$ is the smallest strongly regular equivalence such that the quotient $H / \gamma^{*}$ is a commutative semigroup (resp., a commutative group). The interested reader can find all relevant definitions, many properties and applications of fundamental relations, even in more abstract contexts, also in $[1,4,9,12,16,21]$.

\section{Simple and 0-simple semihypergroups}

In this section we recall the definition of simple and 0-simple semihypergroups; furthermore, we show some examples of such structures that we shall use in the subsequent section. The concept of simple semigroup is well known and widely studied in the framework of semigroup theory [17]. Recently, this concept has been extended to semihypergroup theory [18].

Definition 2.1 A semihypergroup $H$ is simple if $H x H=H$ for all $x \in H$.

If $H$ is a semihypergroup, an element $0 \in H$ such that $0 x=0$ (resp., $x 0=0$ ) for all $x \in H$ is called left zero scalar element (resp., right zero scalar element) of $H$. If 0 is both left and right zero scalar element, then 0 is called zero scalar.

Definition 2.2 A semihypergroup $H$ with a zero scalar element is called 0-simple if $H x H=H$ for all $x \in H-\{0\}$.

We remark that the definitions of simple and 0-simple semihypergroup as given by Definition 2.1 and Definition 2.2 are equivalent to the ones usually introduced by means of the ideals of the semihypergroup, see e.g., $[17,18]$.

Our first examples are immediate:

1. All left- or right-reproducible semihypergroups are simple. Indeed, we have

$$
H x H=H H=\cup_{a \in H} H a=H .
$$

As a consequence, not only all hypergroups are simple semihypergroups, but also all finite semihypergroups of type $U$ on the right are simple, because they are left-reproducible $[8,9,13]$.

2. The following example mirrors a construction of the so-called $K_{H}$-semihypergroups given in [5]: If $H$ is a simple semihypergroup and $\mathfrak{A}=\left\{A_{x}\right\}_{x \in H}$ is a family of nonempty and pairwise disjoint sets, then the set $K=\cup_{x \in H} A_{x}$ is a simple semihypergroup with respect to the hyperproduct $a \circ b=\cup_{z \in x y} A_{z}$, for $a \in A_{x}$ and $b \in A_{y}$.

3. If $(H, \circ)$ and $(K, \bullet)$ are two simple semihypergroups, then the cartesian product $H \times K$ is a simple semihypergroup with respect to the hyperoperation $\otimes$ naturally defined as

$$
(x, y) \otimes(z, w)=(x \circ z) \times(y \bullet w) .
$$

We remark that, in this case, if $H$ is only left-reproducible (but not right-reproducible) and $K$ is only rightreproducible (but not left-reproducible), then $H \times K$ is a simple semihypergroup which is neither left- nor right-reproducible.

4. A more significant example of simple semihypergroup which is neither left- nor right-reproducible is described by the following hyperproduct table:

\begin{tabular}{c|ccccc} 
& $a$ & $b$ & $c$ & $d$ & $e$ \\
\hline$a$ & $a$ & $b$ & $a$ & $b$ & $b$ \\
$b$ & $a$ & $b$ & $a$ & $b$ & $b$ \\
$c$ & $c$ & $d, e$ & $c$ & $d, e$ & $d, e$ \\
$d$ & $c$ & $d, e$ & $c$ & $d, e$ & $d, e$ \\
$e$ & $c$ & $d, e$ & $c$ & $d, e$ & $d, e$
\end{tabular}

In fact, it is apparent that this semihypergroup cannot be obtained as a direct product of smaller semihypergroups. 
The semihypergroup (1) has a remarkable property: All its subsemihypergroups are simple. This fact suggests the following definition, which will be developed in subsequent sections:

Definition 2.3 A semihypergroup $H$ is said strongly simple if it fulfills the following conditions:

1. $H$ and all subsemihypergroups of $H$ are simple;

2. there exist $x, y \in H$ such that $H x \neq H$ and $y H \neq H$.

We remark that strongly simple semihypergroups cannot have left or right zero scalar elements. Indeed, we have the following result:

Proposition 2.4 If $H$ is a simple semihypergroup and $0 \in H$ is a right zero scalar element (resp., left zero scalar element), then $H$ is right-reproducible (resp., left-reproducible).

Proof Since $H$ is simple we have $H=(H 0) H=0 H$. Moreover, for all $a \in H$, we have $a H=a(0 H)=$ (a0) $H=0 H=H$, hence $H$ is right-reproducible. The case when 0 is a left zero scalar is treated analogously.

As an immediate consequence, we obtain the following claim, which will be useful in subsequent arguments:

Corollary 2.5 Let $H$ be a simple semihypergroup that is not right-reproducible (resp., left-reproducible), and let $K \subset H$ be a proper subsemihypergroup. If $a \in K$ is a right (resp., left) zero scalar element of $K$, then $(H-K) a \not \subset K($ resp., $a(H-K) \not \subset K)$.

Proof If $(H-K) a \subset K$ then, using the identity $a^{2}=\{a\}$ we obtain

$$
H a=((H-K) \cup K) a=(H-K) a \cup K a=(H-K) a^{2} \cup K a \subset K a \cup K a=\{a\} .
$$

As a consequence, $H a=\{a\}$ and $a$ is a right zero scalar element of $H$. Hence, by Proposition 2.4, $H$ is right-reproducible, which is a contradiction.

In what follows, we consider two more examples of simple semihypergroups, which deserve some attention. The first example is an extreme generalization of Rees construction [17]. In the second example we provide a list of all simple semihypergroups having order 2, apart of isomorphisms. Some of these semihypergroups will be exploited in the forthcoming section to construct simple semihypergroups having order 3 , whose fundamental relation $\beta$ is not transitive.

Example 2.6 Let $H$ be a hypergroup, let $\mathfrak{A}=\left\{A_{i}\right\}_{i \in I}$ and $\mathfrak{B}=\left\{B_{j}\right\}_{j \in J}$ be two families of nonempty and pairwise disjoint sets, and let $\varphi: J \times I \mapsto \mathcal{P}^{*}(H)$. Introduce the notations $A=\cup_{i \in I} A_{i}, B=\cup_{j \in J} B_{j}$, $E=A \times H \times B$ and $\varphi(h, k)=P_{h k}$ for all $(h, k) \in J \times I$. On the set $E$ we can define the following hyperproduct:

$$
\left.\begin{array}{l}
(a, x, b) \in A_{i} \times H \times B_{j} \\
\left(a^{\prime}, y, b^{\prime}\right) \in A_{k} \times H \times B_{h}
\end{array}\right\} \Longrightarrow(a, x, b) \circ\left(a^{\prime}, y, b^{\prime}\right)=A_{i} \times\left(x P_{j k} y\right) \times B_{h} .
$$

This hyperproduct is associative. Indeed, for any triple $\left(a^{\prime \prime}, z, b^{\prime \prime}\right) \in A_{r} \times H \times B_{s}$, in the hypergroup $H$ we have $\left(x P_{j k} y\right) P_{h r} z=x P_{j k}\left(y P_{h r} z\right)$ and for the hyperproduct in $E$ it holds

$$
\begin{aligned}
{\left[(a, x, b) \circ\left(a^{\prime}, y, b^{\prime}\right)\right] \circ\left(a^{\prime \prime}, z, b^{\prime \prime}\right) } & =\left[A_{i} \times\left(x P_{j k} y\right) \times B_{h}\right] \circ\left(a^{\prime \prime}, z, b^{\prime \prime}\right) \\
& =A_{i} \times\left(x P_{j k} y\right) P_{h r} z \times B_{s}
\end{aligned}
$$

and moreover

$$
\begin{aligned}
(a, x, b) \circ\left[\left(a^{\prime}, y, b^{\prime}\right) \circ\left(a^{\prime \prime}, z, b^{\prime \prime}\right)\right] & =(a, x, b) \circ\left[A_{k} \times\left(y P_{h r} z\right) \times B_{s}\right] \\
& =A_{i} \times x P_{j k}\left(y P_{h r} z\right) \times B_{s} .
\end{aligned}
$$

Hence $\left[(a, x, b) \circ\left(a^{\prime}, y, b^{\prime}\right)\right] \circ\left(a^{\prime \prime}, z, b^{\prime \prime}\right)=(a, x, b) \circ\left[\left(a^{\prime}, y, b^{\prime}\right) \circ\left(a^{\prime \prime}, z, b^{\prime \prime}\right)\right]$ and the hyperproduct is associative. Furthermore, for any fixed pair $(s, r) \in J \times I$ and for any elements $\bar{b} \in B_{s}$ and $\bar{a} \in A_{r}$, from equation $H P_{s i} x P_{j r} H=H$ we obtain that there exists a pair $(w, \bar{w}) \in H \times H$ such that $y \in w P_{s i} x P_{j r} \bar{w}$. As a consequence, $\left(a^{\prime}, y, b^{\prime}\right) \in\left(a^{\prime}, w, \bar{b}\right) \circ(a, x, b) \circ\left(\bar{a}, \bar{w}, b^{\prime}\right)$. In conclusion, we have proven that $E \circ(a, x, b) \circ E=E$ for all $(a, x, b) \in E$, that is, $E$ is a simple semihypergroup. 
The preceding example gives us a very general technique to construct simple semihypergroups; various known constructions can be considered as particular cases, where all the sets in the families $\mathfrak{A}$ and $\mathfrak{B}$ are singletons, so that we can identify $\mathfrak{A}$ and $\mathfrak{B}$ with the index sets $I$ and $J$, respectively. In that case, we shall say that $E$ is a $(H, I, J, \varphi)$-semihypergroup.

1. If $H$ is a group and the map $\varphi$ is single-valued, then the $(H, I, J, \varphi)$-semihypergroup is a semigroup known as Rees matrix semigroup, see [17, §3.3].

2. If $H$ is a regular hypergroup (see e.g., [18] for the definition of regular hypergroup) and the map $\varphi$ is single-valued, then the $(H, I, J, \varphi)$-semihypergroup is the Rees matrix semihypergroup introduced in [18].

3. If $|I|=|J|=1$, then the $(H, I, J, \varphi)$-semihypergroup $E=I \times H \times J$ is a hypergroup, whatever is $\varphi$. Indeed, in the paper [10] it is shown that for any nonempty subset $A$ of a hypergroup $H$, the hyperproduct $o_{A}$ defined as

$$
\forall(x, y) \in H^{2}, \quad x \circ_{A} y=x A y
$$

is associative and $\left(H, \circ_{A}\right)$ is a hypergroup. Now, if $I=\{i\}, J=\{j\}$, and $A=\varphi(j, i)$, then Equation (2) becomes $(i, x, j) \circ(i, y, j))=\{i\} \times x A y \times\{j\}$, whence the map $f: E \mapsto H$ such that $f(i, x, j)=x$ is an isomorphism between $(E, \circ)$ and $\left(H, \circ_{A}\right)$.

Example 2.7 It is not difficult to prove (by an exhaustive procedure) that, apart of isomorphisms, all simple semihypergroups of order 2 are the following:

1. The eight hypergroups of order 2 , see $[6,20]$;

2. The two semigroups

$$
\begin{aligned}
& \begin{array}{l|lll|ll}
x & y & & x & y \\
\hline x & x & y & x & x & x
\end{array}
\end{aligned}
$$

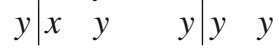

3. The following semihypergroups:



Observe that the four semihypergroups (4) are not $(H, I, J, \varphi)$-semihypergroups. Indeed, all $(H, I, J, \varphi)$ semihypergroups of order 2 fall into the following cases:

1. $|H|=|J|=1$ and $|I|=2$;

2. $|H|=|I|=1$ and $|J|=2$;

3. $|H|=2$ and $|I|=|J|=1$.

In the first two cases the resulting $(H, I, J, \varphi)$-semihypergroup is isomorphic to one of the two semigroups in (3), while in the last case it is a hypergroup.

\section{The relation $\beta$ in simple semihypergroups}

The recent paper [13] has solved the problem of the existence of semihypergroups of type $U$ on the right where the fundamental relation $\beta$ is not transitive. Indeed, in that paper the author exhibits a semihypergroup of type $U$ on the right having cardinality 9 where $\beta$ is not transitive, and moreover, proves that in all semihypergroups of type $U$ on the right having cardinality less than $9 \beta$ is always transitive. Analogous problems arise for the class of strongly simple semihypergroups. In fact, in this section we will prove that there exist exactly 10 simple semihypergroups having order 3, apart of isomorphisms, where the relation $\beta$ is not transitive. Their subsemihypergroups are simple, hence they fulfil the first condition of Definition 2.3; by the way, they are not strongly simple because they are either left- or right-reproducible.

Now, since in all semihypergroups having order 2 the relation $\beta$ is transitive, it is interesting to determine the minimal cardinality for which there exist a strongly simple semihypergroup where the relation $\beta$ is not transitive. This problem will be solved by the forthcoming Theorem 4.4. 
Remark 3.1 If $H$ is a semihypergroup such that $\beta$ is not transitive, then there exists a triple $(a, b, c)$ of distinct elements of $H$ such that $a \beta b, a \beta c$ but $(b, c) \notin \beta$. Obviously, in this case, for any integer $k \geq 1$ and for any $k$-uple $\left(z_{1}, z_{2}, \ldots, z_{k}\right)$ of elements in $H$ we have $\prod_{i=1}^{k} z_{i} \neq H$. Moreover, there exist two hyperproducts $P=\prod_{i=1}^{n} x_{i}$ and $Q=\prod_{i=1}^{m} y_{i}$ such that $\{a, b\} \subset P$ and $\{a, c\} \subset Q$. Now, if we suppose that $H=\{a, b, c\}$ then we have $P=\{a, b\}, Q=\{a, c\}$ and moreover

$$
\begin{array}{ll}
\left.\pi_{1}\right) & P P=a a \cup a b \cup b a \cup b b ; \\
\left.\pi_{2}\right) & P Q=a a \cup a c \cup b a \cup b c ; \\
\left.\pi_{3}\right) & Q P=a a \cup a b \cup c a \cup c b ; \\
\left.\pi_{4}\right) & Q Q=a a \cup a c \cup c a \cup c c .
\end{array}
$$

Furthermore, it holds $H H=H$. Indeed, if this is not the case, the set $H H$ is contained into one of the sets $\{a, b\},\{a, c\}$ or $\{b, c\}$; as a consequence, we would obtain the contradiction $(a, b) \notin \beta$ or $(a, c) \notin \beta$. Finally, we observe that $a a=\{a\}$. Indeed, if we suppose that $b \in a a$, since $c \in H=H H=P P \cup P Q \cup Q P \cup Q Q$, by way of $\left.\left.\pi_{1}\right), \pi_{2}\right), \pi_{3}$ ) and $\pi_{4}$ ) we obtain the contradiction $b \beta c$. Analogously, we prove that $c \notin a a$.

The forthcoming results are preliminary to the construction of simple semihypergroups having order 3 and $\beta$ not transitive.

Lemma 3.2 Let $H=\{a, b, c\}$ be a simple semihypergroup such that $a \beta b, a \beta c$ and $(b, c) \notin \beta$. In the notations of Remark 3.1, we have:

1. $a a=\{a\}$

2. $a b \neq\{b\}$ and $a c \neq\{c\}$;

3. $c \notin P P$;

4. $b \notin Q Q$;

5. $a b=\{a\} \Longleftrightarrow a c=\{a\}$;

\section{Proof}

1. This fact has been shown in Remark 3.1.

2. Let us suppose by absurd that $a b=\{b\}$. Hence, by the preceding point together with $\left.\pi_{1}\right)$ and $\pi_{3}$ ), we have $b a \cup b b \cup c a \cup c b \subset\{a, b\}$. Hence $H a \cup H b \subset\{a, b\}$. Moreover, for any $x \in H$ we obtain $c \in x c$, otherwise for some $x \in H$ we have $x c \subset\{a, b\}$ and we arrive at the contradiction $H=H x H \subset H\{a, b\}=$ $H a \cup H b \subset\{a, b\}$. Hence $x c \in\{\{c\},\{a, c\}\}$ for all $x \in H$ and, in particular, $H c \subset\{a, c\}$.

From $c \in b c, b a \subset\{a, b\}$ and $\left.\pi_{2}\right)$, we derive $b a=\{a\}$. Analogously, from $c \in a c, c a \subset\{a, b\}$ and $\left.\pi_{4}\right)$, we derive $c a=\{a\}$. Furthermore, we have $c b=c(a b)=(c a) b=a b=\{b\}$ and $b b=b(a b)=(b a) b=$ $a b=\{b\}$. Hence $H a=\{a\}, H b=\{b\}$ and $H c \subset\{a, c\}$. This implies that no hyperproduct contains $\{a, b\}$, thus contradicting the hypothesis $a \beta b$. By reversing the role of $b$ and $c$, we prove that $a c \neq\{c\}$.

3. We prove the claim by showing that $c \notin a b \cup b a \cup b b$.

Let us suppose by absurd that $c \in a b$. Firstly, we prove that $b a=c a=\{a\}$. Indeed, by $\left.\pi_{1}\right)$ and $\pi_{3}$ ), one has $b a \cup b b \cup c a \cup c b \subset\{a, c\}$. Moreover, $c \notin b a$. Actually, if $c \in b a$ then by $\left.\pi_{2}\right)$ we obtain the inclusion $a c \cup b c \subset\{a, c\}$. As a consequence $b \in c c$ since $H=H H=\{a, c\} \cup c c$. This fact implies

$$
b \in c c \subset c(a b)=(c a) b \subset\{a, c\} b=a b \cup c b \subset\{a, c\},
$$

which is impossible. Hence $b a=\{a\}$.

Analogously we have $c \notin c a$. Indeed, if $c \in c a$ then by $\left.\pi_{4}\right)$ we obtain the inclusion $a c \cup c c \subset\{a, c\}$. As a consequence $b \in b c$ since $H=H H=\{a, c\} \cup b c$. This fact implies $b \in b c \subset b(a b)=(b a) b=a b \subset$ $\{a, c\}$, which is impossible. Hence $c a=\{a\}$.

From $c \in a b=(b a) b=b(a b) \subset b\{a, c\}=b a \cup b c=\{a\} \cup b c$ we obtain $c \in b c$. Hence, by $\left.\pi_{2}\right)$ it follows that $a c \subset\{a, c\}$ and moreover $H=(H a) H=a H \subset\{a, c\}$, which is absurd. Hence we conclude that $c \notin a b$.

By reversing the roles of $a$ and $b$ we obtain $c \notin b a$.

Finally we prove that $c \notin b b$. Indeed, if $c \in b b$, by 1) and $\left.\pi_{1}\right)$ we have $a a=a b=b a=\{a\}$ and moreover

$$
a c \subset a(b b)=(a b) b=a b=\{a\},
$$

whence $a c=\{a\}$. By exploiting the hyperproduct $b b a$ we also get $c a=\{a\}$. This fact would imply that $a$ is a zero element in $H$ but this is impossible since $H$ is simple. Hence $c \notin P P$. 
4. The proof goes analogously as in the preceding point by interchanging the role of $b$ and $c$ and by exploiting appropriately the conditions $\pi_{i}$ ) for $i=1,2,3,4$.

5. Let $a b=\{a\}$. From the preceding point we obtain $a c \cup c a \cup c c \subset\{a, c\}$. Now if we suppose $c \in a c$, by $\left.\pi_{2}\right)$ and 3., we obtain $b a=\{a\}$ and $b c \subset\{a, c\}$. Hence $H=H(a H)=H\{a, c\}=H a \cup H c \subset\{a, c\}$ which is absurd. So $c \notin a c$ and we conclude $a c=\{a\}$.

The reverse implication $a c=\{a\} \Rightarrow a b=\{a\}$ can be shown analogously by reversing the role of $b$ and $c$ and by applying $\pi_{3}$ ) and the preceding point.

Lemma 3.3 Let $H=\{a, b, c\}$ be a simple semihypergroup such that $a \beta b, a \beta c$ and $(b, c) \notin \beta$. If $a b=\{a\}$ then $b \in b a \cap b b$ and $c \in c a \cap c c$.

Proof By hypothesis and parts 3 and 5 of Lemma 3.2, we have $a b=a c=\{a\}$ and $b a \subset\{a, b\}$. If we suppose that $b a=\{a\}$ then we obtain $H=H(a H)=H a=\{a\} \cup c a$. As a consequence, $\{b, c\} \subset c a$ whence $b \beta c$, a contradiction.

Hence $b \in b a$. Furthermore, if $b b=\{a\}$ then we obtain $\{a\}=a b=(b b) b=b(b b)=b a$ which is impossible. Hence $b \in b a \cap b b$.

The second part of the claim is shown analogously by interchanging the role of $b$ and $c$.

Proposition 3.4 Let $H=\{a, b, c\}$ be a simple semihypergroup such that $a \beta b, a \beta c$ and $(b, c) \notin \beta$. Then, the two sets $P=\{a, b\}$ and $Q=\{a, c\}$ are simple subsemihypergroups of $H$.

Proof Hereafter we prove the claim for $P$. The proof for $Q$ is completely analogous.

By point 3 of Lemma 3.2 we have $P P \subset P$. If $|P P|=1$, by part 1 of Lemma 3.2 we have $a a=a b=$ $b a=\{a\}$ and this fact is in contradiction with Lemma 3.3. Hence $P P=P=\{a, b\}$. Now, for part 2 of Lemma 3.2 we have $a b \in\{\{a\},\{a, b\}\}$. If $a b=\{a, b\}$ then it follows immediately that $P a P=P b P=P$. On the other hand, if $a b=\{a\}$ then by Lemma 3.2 and Lemma 3.3 we obtain $a a=a b=\{a\}$ and $b \in b a \cap b b$. Thus $a \in(a b) a$ and $b \in(b b) a$. As a consequence, we get $P a P=P(a P)=P a=a a \cup b a=P$ and $P \subset(a b) a \cup(b b) a \subset P b P \subset P$. Hence, also in this case we obtain $P a P=P b P=P$.

In what follows, we will describe the isomorphism classes of the simple semihypergroups having order 3 where the relation $\beta$ is not transitive. By Remark 3.1, we can limit ourselves to the case where $H=\{a, b, c\}$ is a simple semihypergroup such that $a \beta b, a \beta c$ and $(b, c) \notin \beta$ Moreover, by Lemma 3.2 and Proposition 3.4, we can restrict our analysis to the cases $a b=\{a, b\}$ and $a b=\{a\}$.

\subsection{First step: $a b=\{a, b\}$}

If we assume that $a b=\{a, b\}$ then we can deduce that

$$
a a=b a=\{a\}, a b=\{a, b\} \text { and } a a=c a=\{a\}, a c=\{a, c\} .
$$

Indeed, by $\pi_{3}$ ), one has $c \notin c a$ otherwise $Q P=H$ and $b \beta c$. Moreover, since $b \notin Q Q$, we have $b \notin c a$ and thus $c a=\{a\}$. Furthermore, $a c \neq\{a\}$, otherwise $a$ is a zero element in $Q$ and $Q$ is not simple. Hence, by point 2 of Lemma 3.2, we obtain $a c=\{a, c\}$. Finally, by $\left.\pi_{2}\right)$, we get $b a=\{a\}$.

Now, from Example 2.7 we know that, apart of isomorphisms, there exist only two simple semihypergroups $S_{i}=\{x, y\}$, for $i=1,2$, such that $x x=y x=\{x\}$ and $x y=\{x, y\}$. Their multiplicative tables are the following:

$$
S_{1}: \begin{array}{l|lc}
x & x & y \\
y & x & x, y
\end{array} \quad S_{2}: \begin{array}{l|ll}
x & x & y \\
y & x & x, y \\
x & x, y
\end{array}
$$

Consequently, from conditions (5) we obtain that the two simple subsemihypergroups $P=\{a, b\}$ and $Q=$ $\{a, c\}$ must be isomorphic to $S_{1}$ or $S_{2}$. Hence, the only possible cases in the present step are contained in the following partial tables:

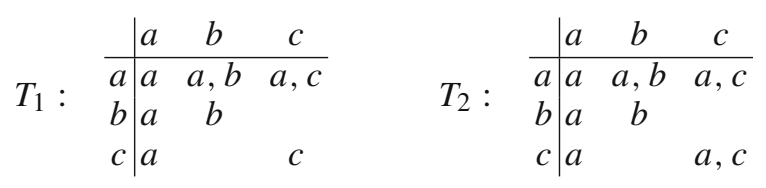




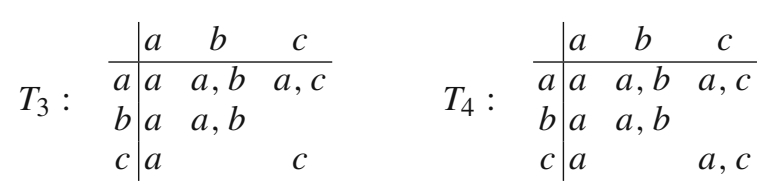

Now, we observe that the hyperproducts $\pi_{2}$ ) and $\pi_{3}$ ) in Remark 3.1 imply respectively $b c \subset\{a, c\}$ and $c b \subset\{a, b\}$. Moreover, $b c \neq\{a\}$ otherwise one has $c \in a c \subset(a b) c=a(b c)=a a=\{a\}$. Analogously $c b \neq\{a\}$ otherwise $b \in a b \subset(a c) b=a(c b)=a a=\{a\}$. Hence

$$
b c \in\{\{c\},\{a, c\}\} \text { and } c b \in\{\{b\},\{a, b\}\} .
$$

Finally, from (9) we get the following implications:

1. $b b=\{a, b\} \Rightarrow b c=\{a, c\}$. Otherwise $b c=\{c\}$ and the product $b b c$ leads to the contradiction $\{c\}=\{a, c\}$.

2. $c b=\{b\} \Rightarrow c c=\{c\}$. Indeed, if $c c=\{a, c\}$ the product $c c b$ leads to the contradiction $\{b\}=\{a, b\}$.

3. As a consequence, the partial table $T_{4}$ is completed by the simple semihypergroup

$$
\begin{array}{rl|lll}
H_{1}: & & a & b & c \\
a & a & a, b & a, c \\
b & a & a, b & a, c \\
c & a & a, b & a, c
\end{array}
$$

The partial table $T_{3}$ leads to the two simple semihypergroups

$$
H_{2}: \begin{array}{c|lcc}
a & b & c \\
\hline a & a & a, b & a, c \\
b & a & a, b & a, c \\
c & a & b & c
\end{array} \quad H_{3}: \begin{array}{rl|lcc}
\quad & a & a & a, b & a, c \\
b & a & a, b & a, c \\
c & a & a, b & c
\end{array}
$$

Furthermore, the partial table $T_{2}$ can be completed as

$$
H_{2}^{\prime}: \begin{array}{c|lcc}
\mid & a & b & c \\
\hline a & a & a, b & a, c \\
b & a & b & c \\
c & a & a, b & a, c
\end{array} \quad H_{3}^{\prime}: \begin{aligned}
& a \\
& b
\end{aligned} \quad \begin{array}{llll}
a & a, b & a, c \\
c & a & a, b & a, c
\end{array}
$$

that are two simple semihypergroups isomorphic to $\mathrm{H}_{2}$ and $\mathrm{H}_{3}$ respectively.

4. $b b=c b=\{b\} \Rightarrow b c=\{c\}$. Otherwise $b c=\{a, c\}$ and the product $b c b$ leads to the contradiction $\{b\}=\{a, b\}$.

5. If $b b=\{b\}$ and $c b=\{a, b\}$ then $b c=\{c\} \Rightarrow c c=\{a, c\}$. Otherwise $c c=\{c\}$ and the product $c b c$ leads to $\{c\}=\{a, c\}$.

6. As a consequence, the partial table $T_{1}$ can be completed only by the following simple semihypergroups:

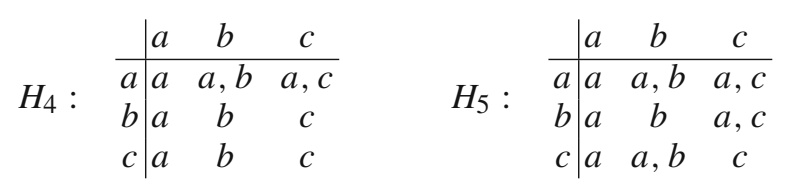

So far, we have obtained five simple semihypergroups having order 3 where the relation $\beta$ is not transitive. These semihypergroups are pairwise non isomorphic. Furthermore, they are not strongly simple, because they are right-reproducible. 


\subsection{Second step: $a b=\{a\}$}

We begin with the following remark: If $(H, \circ)$ is a semihypergroup then $H$ is also a semihypergroup with respect to the hyperproduct $\bullet$ defined as $x \bullet y=y \circ x$ for all $x, y \in H$. In what follows, the semihypergroup $(H, \bullet)$ will be called the transposed of $(H, \circ)$ and will be denoted simply by $H^{T}$. Clearly, the use of that term is motivated by the fact that, in the finite case, the multiplicative table of $H^{T}$ is obtained by transposing the multiplicative table of $H$. Obviously, if $H$ and $K$ are two isomorphic semihypergroups then also $H^{T}$ and $K^{T}$ are isomorphic. Furthermore, we note that in general $H$ is not isomorphic to $H^{T}$. For example, it is easy to check that for $i=1, \ldots, 5$ the transposed semihypergroups $H_{i}^{T}$ are not isomorphic respectively to the semihypergroups $H_{i}$ obtained in Eqs. (10), (11) and (13). Finally, we observe that the relation $\beta$ is transitive in $H$ if and only if it is transitive in $H^{T}$. Moreover, $H$ is simple (resp., left-reproducible) if and only if $H^{T}$ is simple (resp., right-reproducible).

In the following we will show that, apart of isomorphisms, the semihypergroups $H_{i}^{T}$ for $i=1, \ldots, 5$ are the only simple semihypergroups having order 3 where the relation $\beta$ is not transitive and $a a=a b=\{a\}$. Indeed, if $K$ is a semihypergroup verifying these conditions, then the simple subsemihypergroup $P=\{a, b\}$ is isomorphic to one of the two semihypergrups

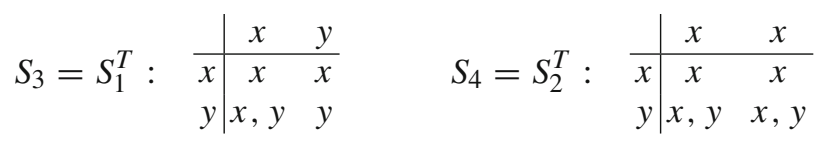

In particular, in $K$ one has $b a=\{a, b\}$. This fact implies that in $K^{T}$ one has $a b=\{a, b\}$. As a consequence, there exists $i \in\{1, \ldots, 5\}$ such that $K^{T} \cong H_{i}$. Hence $K \cong\left(K^{T}\right)^{T} \cong H_{i}^{T}$. In conclusion, we can state the following result:

Theorem 3.5 Apart of isomorphisms, there exist 10 simple semihypergroups having order 3 where the relation $\beta$ is not transitive. These semihypergroups are $\mathrm{H}_{1}, \mathrm{H}_{2}, \mathrm{H}_{3}, \mathrm{H}_{4}, \mathrm{H}_{5}$ and their respective transposed semihypergroups.

\section{Strongly simple extensions}

The ten semihypergroups in Theorem 3.5 fulfil the first condition in Definition 2.3 but not the second one. In fact, for $i=1, \ldots, 5$ the semihypergroup $H_{i}$ is right-reproducible, while $H_{i}^{T}$ is left-reproducible. This fact lead us to the problem of determining the least order for a strongly simple semihypergroup whose relation $\beta$ is not transitive. In this section we will prove that this number is 4 , see Theorem 4.4. The proof consists in the construction of suitable extensions having order 4 of the semihypergroups $H_{i}$. The reader can find various extension techniques exploited in semigroup and hypergroup theory in $[14,15]$.

Lemma 4.1 Let $H$ be a semihypergroup, and let $K$ be a subsemihypergroup of $H$ such that $H=K \cup\{d\}$. If $a \in K$ is a right zero scalar element of $K$ and $d \in d a$, then

1. $H d \subset\{a\} \cup d a$;

2. $d \in d y$, for all $y \in K$.

Proof

1) For any $x \in H$ let $A=x d \cap K$ and $B=x d \cap\{d\}$. Using the inclusions $A \subset K$ and $B \subset\{d\}$ we obtain $x d \subset x d a=(A \cup B) a=A a \cup B a \subset K a \cup d a=\{a\} \cup d a$.

2) Let $y \in K$. If we suppose that $d y \subset K$ then we arrive at the contradiction $d \in d a=d(y a)=(d y) a \subset$ $K a=\{a\}$. Hence $d \in d y$.

Lemma 4.2 Let $H=\{a, b, c, d\}$ be a strongly simple semihypergroup such that $a \beta b, a \beta c$ and $(b, c) \notin \beta$. If $K=\{a, b, c\}$ is a subsemihypergroup isomorphic to a simple semihypergroup $H_{i}$ as in Theorem 3.5, for some $i=1, \ldots 5$, then we have

1. $d a=\{a, d\}$;

2. $\{b, d\} \subset d b$ and $\{c, d\} \subset d c$;

3. $a d=b d=c d=\{a\}$;

4. $d b \in\{\{b, d\},\{a, b, d\}\}$ and $d c \in\{\{c, d\},\{a, c, d\}\}$; 
5. $d d \in\{\{d\},\{a, d\}\}$.

Proof We start by observing that, by hypotheses, one has

$$
K a=\{a\}, \quad a b=\{a, b\}, \quad a c=\{a, c\}, \quad b \in b b \cap c b, \quad c \in b c \cap c c .
$$

1. We prove the first claim by proceeding in three steps:

I) $d a \not \subset K$;

II) $b \notin d a$ and $c \notin d a$;

III) $d a \neq\{d\}$.

I) The claim follows immediately from Corollary 2.5, since we have $H-K=\{d\}$.

II) If $b \in d a$ then one has $c \in b c \subset(d a) c$ and $b \in d a \subset d(a c)$. We arrive at the contradiction $b \beta c$. The case $c \in d a$ is treated analogously.

III) Let us suppose by absurd that $d a=\{d\}$. This leads to $d b=d c=\{d\}$ and consequently

$$
d K=\{d\} \text { and } d H=\{d\} \cup d d .
$$

Indeed, if there exists $x \in\{b, c\}$ such that $d x \cap K \neq \emptyset$, then we arrive at the contradiction $\{a\}=(d x \cap K) a \subset$ $(d x) a=d(x a)=d a=\{d\}$.

Furthermore, by Lemma 4.1(1), we have $a d \subset d a \cup\{a\}=\{a, d\}$. We are led to the following three subcases: (i) $a d=\{a\}$; (ii) $a d=\{d\}$; (iii) $a d=\{a, d\}$.

(i) If $a d=\{a\}$ then $d d=(d a) d=d(a d)=d a=\{d\}$. Hence $d H=\{d\}$ and $d$ is a left zero scalar element of $H$. This is in contradiction with Proposition 2.4 since $H$ is strongly simple by hypothesis.

(ii) If $a d=\{d\}$, by hypotheses and Equation (15), we have $b d=b(a d)=(b a) d=a d=\{d\}$. Analogously, we also have $c d=c(a d)=(c a) d=$ $a d=\{d\}$. Thus $H d=\{d\} \cup d d$. Since $H$ is simple, using (16) we obtain

$$
H=H(d H)=H(\{d\} \cup d d)=H d \cup H d d=\{d\} \cup d d \cup d d d .
$$

Now, if $d \in d d$ then we obtain $d \in d d \subset d d d$ and $H=d d d$. This would imply $b \beta c$, a contradiction with the hypotheses. On the other hand, if $d \notin d d$ then $d d \subset K$ and $d d d \subset d K=\{d\}$ by (16). As a consequence, $d d d=\{d\}$ whence $K=d d$. This would imply again $b \beta c$.

(iii) Let $a d=\{a, d\}$. Since by hypothesis $d a=\{d\}$, from Lemma 4.1(1) we have $b d \cup c d \subset\{a, d\}$ and consequently $H d=\{a, d\} \cup d d$. Hence, since $H$ is simple, using (16) we have

$$
H=H(d H)=H(\{d\} \cup d d)=H d \cup H d d=\{a, d\} \cup d d \cup(\{a, d\} \cup d d) d=\{a, d\} \cup d d \cup d d d .
$$

By proceeding as in subcase (ii), we obtain either $H=\{a, d\} \cup d d d$ or $H=\{a, d\} \cup d d$ depending on whether $d \in d d$ or $d d \subset K$. In both cases we obtain the contradiction $b \beta c$.

Finally, from I), II) and III) we conclude that $d a=\{a, d\}$.

2. From Eq. (15) we know that $a b=\{a, b\}$. Moreover, by point 1.) we have

$$
b \in a b \subset\{a, d\} b=(d a) b=d(a b)=d\{a, b\}=d a \cup d b=\{a, d\} \cup d b .
$$

Consequently $b \in d b$. Analogously, from $a c=\{a, c\}$ one also get $c \in d c$. Finally, by Lemma 4.1(2), one has $d \in d b \cap d c$, and the claim follows.

3. From points 1.) and 2.) we have $d H=H$. Moreover, by Lemma 4.1(1) and point 1.), we have $x d \subset\{a, d\}$ for all $x \in H$. Owing to the fact that $K$ is right-reproducible, if we suppose by absurd that $d \in x d$ for all $x \in K$, then it follows that $x H=x(K \cup\{d\})=x K \cup x d=K \cup x d=H$, that is, $H$ is right-reproducible. This is in contradiction with the hypothesis that $H$ is strongly simple. Hence, there exists some $x \in K$ such that $x d=\{a\}$. Using (15) we obtain the claim. Indeed we have:

- $a d=(x d) d=x(d d) \subset x\{a, d\}=x a \cup x d=\{a\}$;

- $b d \subset(a b) d=a(b d) \subset a\{a, d\}=a a \cup a d=\{a\}$;

- $c d \subset(a c) d=a(c d) \subset a\{a, d\}=\{a\}$.

Hence $a d=b d=c d=\{a\}$.

4. If $c \in d b$, by point 3.) and (15) we have $\{b, c\} \subset a b \cup a c \subset a b \cup a(d b)=a b \cup(a d) b=a b$ and $b \beta c$, which is absurd. Hence $c \notin d b$. Analogously we can prove that $b \notin d c$. Finally, by point 2.), we also have $d b \in\{\{b, d\},\{a, b, d\}\}$ and $d c \in\{\{c, d\},\{a, c, d\}\}$. 
5. By 1.) and Lemma 4.1(1), we have $d d \subset\{a, d\}$. Moreover $d d \neq\{a\}$ otherwise one has $H d=\{a\}$ by 3.). Consequently, from (15) we get the contradiction $H=(H d) H=a H=a K \cup a d=K$. Hence $d d \in\{\{d\},\{a, d\}\}$ and the proof is complete.

Remark 4.3 Using Lemma 4.2, we can construct various semihypergroups having order 4 where $\beta$ is not transitive. In fact, if we consider as $K=\{a, b, c\}$ any semihypergroup $H_{i}$ as in Theorem 3.5, with $i=1,2,3,4,5$, then we can embed $K$ in a semihypergroup $H=\{a, b, c, d\}$ by extending the hyperproduct of $K$ as follows:

$$
a d=b d=c d=\{a\}, \quad d a=\{a, d\}
$$

The products $d b, d c$ and $d d$, which are constrained by Lemma 4.2, can be chosen from the following tables: If $K \cong H_{1}, H_{3}, H_{5}$ then

\begin{tabular}{|c|c|c|}
\hline$d b$ & $d c$ & $d d$ \\
\hline \hline$\{b, d\}$ & $\{c, d\}$ & $\{d\}$ \\
\hline$\{b, d\}$ & $\{a, c, d\}$ & $\{d\}$ \\
\hline$\{a, b, d\}$ & $\{c, d\}$ & $\{d\}$ \\
\hline$\{a, b, d\}$ & $\{a, c, d\}$ & $\{d\}$ \\
\hline$\{b, d\}$ & $\{c, d\}$ & $\{a, d\}$ \\
\hline$\{a, b, d\}$ & $\{c, d\}$ & $\{a, d\}$ \\
\hline$\{b, d\}$ & $\{a, c, d\}$ & $\{a, d\}$ \\
\hline$\{a, b, d\}$ & $\{a, c, d\}$ & $\{a, d\}$ \\
\hline
\end{tabular}

if $K \cong H_{2}$ then

\begin{tabular}{|c|c|c|}
\hline$d b$ & $d c$ & $d d$ \\
\hline \hline$\{b, d\}$ & $\{c, d\}$ & $\{d\}$ \\
\hline$\{a, b, d\}$ & $\{c, d\}$ & $\{d\}$ \\
\hline$\{a, b, d\}$ & $\{a, c, d\}$ & $\{d\}$ \\
\hline$\{b, d\}$ & $\{c, d\}$ & $\{a, d\}$ \\
\hline$\{a, b, d\}$ & $\{c, d\}$ & $\{a, d\}$ \\
\hline$\{a, b, d\}$ & $\{a, c, d\}$ & $\{a, d\}$ \\
\hline
\end{tabular}

and if $K \cong H_{4}$ then

\begin{tabular}{|c|c|c|}
\hline$d b$ & $d c$ & $d d$ \\
\hline \hline$\{b, d\}$ & $\{c, d\}$ & $\{d\}$ \\
\hline$\{a, b, d\}$ & $\{a, c, d\}$ & $\{d\}$ \\
\hline$\{b, d\}$ & $\{c, d\}$ & $\{a, d\}$ \\
\hline$\{a, b, d\}$ & $\{a, c, d\}$ & $\{a, d\}$ \\
\hline
\end{tabular}

The cases in table (17) are all those possible due to Lemma 4.2. The cases listed in the tables (18) and (19) take into account the following properties, that must be considered when $\mathrm{H}$ extends $\mathrm{H}_{2}$ or $\mathrm{H}_{4}$ :

- If $c b=\{b\}$ then $a \in d c \Rightarrow a \in d b$. Indeed, it holds $a \in a d \subset(d c) b=d(c b)=d b$.

- If $b c=\{c\}$ then $a \in d b \Rightarrow a \in d c$. Indeed, it holds $a \in a c \subset(d b) c=d(b c)=d c$.

By direct verification, the hyperproduct in $H$ as defined by all cases listed in (17), (18) and (19) is associative. Furthermore, the resulting semihypergroups $H$ and their transposed semihypergroups $H^{T}$ are strongly simple and the relation $\beta$ is not transitive. Hence, we obtained the following result:

Theorem 4.4 The least order for which there exists a strongly simple semihypergroup where $\beta$ is not transitive is 4 . 


\section{The relation $\beta$ in 0 -simple semihypergroups}

In semigroup theory, a relevant family is that of 0 -simple semigroups [17]. This concept has been extended in [18] to semihypergroups, see Definition 2.2. Obviously, a 0 -simple semihypergroup is not simple. Hence, the analysis carried out in Sect. 4 (in particular, concerning the transitivity of $\beta$ ) cannot be extended immediately to 0 -simple semihypergroups.

In this section we present all 0 -simple semihypergroups having order 3 where $\beta$ is not transitive, see Theorem 5.6. Owing to Remark 3.1, hereafter we can restrict ourselves to the case where $H=\{a, b, c\}$ has a zero scalar element, $a \beta b, a \beta c$ and $(b, c) \notin \beta$.

Lemma 5.1 If $H=\{a, b, c\}$ is a 0 -simple semihypergroup where $a \beta b, a \beta c$ and $(b, c) \notin \beta$, then the zero element of $H$ is $a$.

Proof Proceeding by absurd, suppose that the zero element of $H$ is $b$. As already shown in Remark 3.1, we have $a a=\{a\}$. Furthermore, by the equalities $\left.\pi_{2}\right)$ and $\left.\pi_{3}\right)$ in the same Remark, we have $a c \cup c a \subset\{a, b\}$.

Now, if $c c \subset\{a, b\}$ then $H H \subset\{a, b\}$, and we obtain $(a, c) \notin \beta$, a contradiction. On the other hand, if $c \in c c$ then, by $\left.\pi_{4}\right)$, one has $b \notin(a c \cup c a)$, otherwise $b \beta c$. Hence $a c=c a=\{a\}$. Finally, we obtain $H=H a H=\{a, b\}$, impossible. This completes the proof.

Lemma 5.2 If $H=\{a, b, c\}$ is a 0 -simple semihypergroup with $a \beta b, a \beta c$ and $(b, c) \notin \beta$, then $x y \neq\{a\}$ for all $x, y \in\{b, c\}$.

Proof We proceed by absurd and suppose that $x y=\{a\}$ for some $x, y \in\{b, c\}$. We split the proof in two cases: 1. $x=y$ and 2. $x \neq y$.

1. Without loss of generality, we can suppose that $x=y=b$. Then we have $H=H b H=(\{a\} \cup c b) H=$ $\{a\} \cup c b c$. Hence $\{b, c\} \subset c b c$ and we arrive at the contradiction $b \beta c$.

2. Without loss of generality, we can suppose that $x=b$ and $y=c$. From Lemma 5.1 we have:

$$
\{a\}=a H=(b c) H=b(c H)=\{a\} \cup b(c b \cup c c) .
$$

Now, if $b \in c b \cup c c$ then necessarily $b b=\{a\}$, and we fall back in the preceding case. On the other hand, if $c b \cup c c \subset\{a, c\}$ then we have $H=H c H=H\{a, c\} \subset\{a, c\}$, impossible.

Proposition 5.3 There exists exactly one 0-simple semihypergroup $H=\{a, b, c\}$ where $\beta$ is not transitive and $b b=\{c\}$. Its hyperproduct table is the following:

\begin{tabular}{c|ccc} 
& $a$ & $b$ & $c$ \\
\hline$a$ & $a$ & $a$ & $a$ \\
$b$ & $a$ & $c$ & $a, b$ \\
$c$ & $a$ & $a, b$ & $a, c$
\end{tabular}

Proof In the stated hypotheses, the zero element cannot be $b$, otherwise we would have $b b=\{b\}$. Moreover, it cannot be $c$ because this would contradict Lemma 5.2. Hence, the zero element is $a$ and, by Lemma 5.1, we have $a \beta b, a \beta c$ and $(b, c) \notin \beta$.

Note that $b c=b(b b)=(b b) b=c b$, hence $b c=c b$. We are led to the following partial table:

$$
\begin{array}{l|lll} 
& a & b & c \\
\hline a & a & a & a \\
b & a & c & X \\
c & a & X &
\end{array}
$$

If $X=\{a, b\}$ then we must have $c c=(b b) c=b(b c)=b\{a, b\}=\{a, c\}$, and the table is completed as claimed. It remains to prove that $X=\{a, b\}$.

Firstly, note that $|c b| \neq 1$. Indeed, if $|c b|=1$ then $|c c|=|c(b b)|=|(c b) b|=1$, since the second column of the hyperproduct table is made of singletons. As a consequence, all hyperproducts reduce to singletons, $H$ is a semigroup and $\beta$ is transitive, a contradiction.

Owing to the hypotheses placed on $\beta$, the remaining cases are $c b=\{a, b\}$ and $c b=\{a, c\}$. If $c b=\{a, c\}$ then $c c=(b b) c=b(b c)=b\{a, c\}=\{a, c\}$, whence $b \notin H H$, impossible. Hence, the only possible case is $b c=\{a, b\}$, which leads to a truly associative hyperproduct, and the proof is complete. 
Lemma 5.4 If $H=\{a, b, c\}$ is a 0-simple semihypergroup with $a \beta b, a \beta c$ and $(b, c) \notin \beta$, then

1. if $b c=\{b\}$ or $c b=\{b\}$ then $c c=\{c\}$,

2. if $b c=\{c\}$ or $c b=\{c\}$ then $b b=\{b\}$.

Proof It is sufficient to prove only the case where $b c=\{b\}$, since the other cases follow by transposition or by interchanging the roles of $b$ and $c$.

In this case we have $\{b\}=(b c) c=b(c c)$. Now, $a \notin c c$ else we obtain the contradiction $\{a\}=b a \subset$ $b(c c)=\{b\}$. If $b \in c c$ then $c c=\{b\}$ since $(b, c) \notin \beta$. As a consequence $b b=\{b\}$ and $c b=c(b c)=(c b) c \subset$ $H c=\{a, b\}$. This implies $H=H H \subset\{a, b\}$, impossible. Finally we must have $c c=\{c\}$ and the proof is complete.

Proposition 5.5 In the same hypotheses of Lemma 5.4, the partial table

$$
\begin{array}{c|ccc} 
& a & b & c \\
\hline a & a & a & a \\
b & a & a, c & \\
c & a & &
\end{array}
$$

can be completed only in the following five ways:

\begin{tabular}{|c|c|c|}
\hline$b c$ & $c b$ & $c c$ \\
\hline \hline$\{b\}$ & $\{b\}$ & $\{c\}$ \\
\hline$\{a, b\}$ & $\{b\}$ & $\{c\}$ \\
\hline$\{b\}$ & $\{a, b\}$ & $\{c\}$ \\
\hline$\{a, b\}$ & $\{a, b\}$ & $\{c\}$ \\
\hline$\{a, b\}$ & $\{a, b\}$ & $\{a, c\}$ \\
\hline
\end{tabular}

Proof If $b c \subset\{a, c\}$ then $H=H b H=H\{a, c\}=\{a\} \cup b c \cup c c$. Hence $b \in c c$ and we arrive at the following contradiction:

$$
b \in c c \subset(b b) c=b(b c) \subset b\{a, c\} \subset\{a, c\} .
$$

Hence $b \in b c$ and we have either $b c=\{b\}$ or $b c=\{a, b\}$. Furthermore, $c b \subset(b b) b=b(b b)=b\{a, c\}=$ $\{a, b\}$ and, by Lemma 5.2, we have either $c b=\{b\}$ or $c b=\{a, b\}$. Whenever $|b c|=1$ or $|c b|=1$, Lemma 5.4 lead us to complete the hyperproduct table as specified in the first three rows of (20). Finally, when $b c=c b=\{a, b\}$ we obtain $c c \subset(b b) c=b(b c)=\{a, c\}$. Again by Lemma 5.2 we have either $c c=\{c\}$ or $c c=\{a, c\}$, and we obtain the last two rows in (20). Associativity of resulting hyperproducts are immediately verified.

At this point, only very few cases are left. Indeed, taking into account the preceding Propositions, it remains to consider when either $b b=\{b\}$ or $b b=\{a, b\}$ and, moreover, either $c c=\{c\}$ or $c c=\{a, c\}$. In fact, the case $c c=\{b\}$ is isomorphic to the one considered in Proposition 5.3, and the case $c c=\{a, c\}$ is isomorphic to the one considered in Proposition 5.5. Furthermore, Lemma 5.4 gives us further restrictions on the aforementioned cases, that can be tackled individually. We refrain from giving explicit proofs, which can be carried out by arguments similar to the ones exploited in the aforementioned Propositions. The conclusion is that, apart of isomorphisms, there are 80 -simple semihypergroups outside the cases considered in Propositions 5.3 and 5.5. Their hyperproduct tables are defined in the following list, neglecting the obvious products of the form $x a=a x=\{a\}$ :

\begin{tabular}{|c|c|c|c|}
\hline$b b$ & $b c$ & $c b$ & $c c$ \\
\hline \hline$\{b\}$ & $\{c\}$ & $\{a, b\}$ & $\{a, c\}$ \\
\hline$\{b\}$ & $\{a, b\}$ & $\{a, c\}$ & $\{c\}$ \\
\hline$\{b\}$ & $\{a, b\}$ & $\{a, c\}$ & $\{a, c\}$ \\
\hline$\{a, b\}$ & $\{a, b\}$ & $\{a, c\}$ & $\{a, c\}$ \\
\hline$\{b\}$ & $\{a, b\}$ & $\{c\}$ & $\{a, c\}$ \\
\hline$\{b\}$ & $\{a, c\}$ & $\{a, b\}$ & $\{c\}$ \\
\hline$\{b\}$ & $\{a, b\}$ & $\{a, c\}$ & $\{a, c\}$ \\
\hline$\{a, b\}$ & $\{a, c\}$ & $\{a, b\}$ & $\{a, c\}$ \\
\hline
\end{tabular}


Note that the semihypergroups defined by the last 4 rows are the transposed semihypergroups of those defined by the first 4 rows. All preceding results in this Section, in particular, Proposition 5.3, Proposition 5.5 and the table (21), can be summarized in the following result:

Theorem 5.6 Apart of isomorphisms, there exist exactly 14 0-simple semihypergroups having order 3 where $\beta$ is not transitive. Their hyperproduct tables are given in Proposition 5.3, Proposition 5.5 and (21).

\section{The relations $\gamma$ and $\gamma^{*}$ in simple and 0 -simple semihypergroups}

The relations $\gamma$ and $\gamma^{*}$ were introduced in [11,12] in the context of hypergroups, in order to characterize the derived hypergroup by means of the notion of strongly regular equivalence. In particular, in [12] a geometric interpretation of $\gamma$ and $\gamma^{*}$ is found, showing their relationships with the concepts of geometric space and polygonal. Subsequently, various authors exploited the concept of geometric space and extended the relation $\gamma$ to other hyperstructures, see e.g., [1,4]. In what follows, we summarize the relationship between semihypergroups and geometric spaces $[4,11,12]$.

A geometric space is a pair $(S, \mathcal{B})$ where $S$ is a nonempty set whose elements are called points, and $\mathcal{B}$ is a family of subsets of $S$, whose elements are called blocks. A polygonal is a $n$-tuple $\left(B_{1}, \ldots, B_{n}\right)$ of blocks such that $B_{i} \cap B_{i+1} \neq \emptyset$ for all $i=1,2, \ldots, n-1$. On $S$ we can define the relations $\sim, \approx$ as follows:

- $x \sim y \Longleftrightarrow x=y$ or there exists $B \in \mathcal{B}$ such that $\{x, y\} \subset B$.

- $x \approx y \Longleftrightarrow x=y$ or there exists a polygonal $\left(B_{1}, \ldots, B_{n}\right)$ such that $x \in B_{1}$ and $y \in B_{n}$.

The relation $\approx$ is the transitive closure of $\sim$. Moreover, if $[x]$ denotes the $\approx$-class of an element $x \in S$ and $B_{1}, B_{2}$ are two blocks in $\mathcal{B}$, then

$$
x \in B_{1} \cup B_{2} \text { and } B_{1} \cap B_{2} \neq \varnothing \quad \Longrightarrow \quad B_{1} \cup B_{2} \subset[x] .
$$

A geometric space is strongly transitive if the family $\mathcal{B}$ is a covering of $S$ and, for all pair $(A, B)$ of blocks in $\mathcal{B}$ one has

$$
A \cap B \neq \emptyset \text { and } x \in B \quad \Longrightarrow \quad \exists C \in \mathcal{B}: A \cup\{x\} \subset C .
$$

We recall from [12] the following theorem:

Theorem 6.1 If the geometric space $(S, \mathcal{B})$ is strongly transitive then $\sim$ is transitive and $\sim$ coincides with $\approx$.

To every semihypergroup $H$ we can associate the geometric space $\left(H, P_{\sigma}(H)\right)$ whose blocks are defined as follows: For all integer $n \geq 1$ and for all $n$-tuple $\left(z_{1}, \ldots z_{n}\right) \in H^{n}$, we set $B\left(z_{1}, z_{2}, \ldots, z_{n}\right)=$ $\cup_{\sigma \in S_{n}} z_{\sigma(1)} \cdots z_{\sigma(n)}$. In particular, $B\left(z_{1}\right)=\left\{z_{1}\right\}$. The blocks in $P_{\sigma}(H)$ have the following properties, see [12]:

1. $B\left(z_{1}, z_{2}, \ldots, z_{n}\right)=B\left(z_{\sigma(1)}, z_{\sigma(2)}, \ldots, z_{\sigma(n)}\right)$, for all $\sigma \in S_{n}$;

2. $z B\left(z_{1}, \ldots, z_{n}\right) z^{\prime} \subset B\left(z, z_{1}, \ldots, z_{n}, z^{\prime}\right)$;

3. If $z_{k} \in x_{1} \cdots x_{m}$ then $B\left(z_{1}, z_{2}, \ldots, z_{n}\right) \subset B\left(z_{1}, \ldots, z_{k-1}, x_{1}, \ldots, x_{m}, z_{k+1}, \ldots, z_{n}\right)$;

4. $B\left(z_{1}, \ldots, z_{n}\right) \cdot B\left(w_{1}, \ldots, w_{m}\right) \subset B\left(z_{1}, \ldots, z_{n}, w_{1}, \ldots, w_{m}\right)$.

Obviously, the relations $\gamma, \gamma^{*}$ are the relations $\sim, \approx$ defined on $H$ arising from the blocks of $P_{\sigma}(H)$. We recall from [12, Thm. 3.4] that if $H$ is a hypergroup then the geometric space $\left(H, P_{\sigma}(H)\right)$ is strongly transitive, thus $\sim$, that is $\gamma$, is transitive. Recently in [13] it was proved that $\gamma$ is transitive even if $H$ is a finite semi-hypergroup of type $U$ on the right. In what follow, we will show that $\gamma$ is transitive is all simple semihypergroups, see Theorem 6.4. We remark that this fact is not necessarily true if $H$ is only 0 -simple. Indeed, for example, the 0 -simple semihypergroup shown in Proposition 5.3 is commutative, thus $\beta=\gamma$, whence $\gamma$ is not transitive. In the next theorem we characterize all 0 -simple semihypergroups whose associated geometric space $\left(H, P_{\sigma}(H)\right)$ is strongly transitive.

Theorem 6.2 If $H$ is a 0 -simple semihypergroup and $\mathfrak{B}_{0}=\left\{B \in P_{\sigma}(H): B \subset \gamma^{*}(0)\right\}$ then the following conditions are equivalent:

1. The geometric space $\left(H, P_{\sigma}(H)\right)$ is strongly transitive.

2. For any block $B \in \mathfrak{B}_{0}$ and for all $x \in \gamma^{*}(0)$ there exists a block $C \in \mathfrak{B}_{0}$ such that $B \cup\{x\} \subset C$. 
Proof $1 . \Rightarrow 2$. By Theorem 6.1 we have that $\gamma$ is transitive. Hence, for all $B \in \mathfrak{B}_{0}$, for all $y \in B$ and for all $x \in \gamma^{*}(0)$ we have $y \gamma x$. As a consequence, there exists a block $B^{\prime} \in P_{\sigma}(H)$ such that $\{y, x\} \subset B^{\prime}$. Since $B \cap B^{\prime} \neq \emptyset$ and the geometric space $\left(H, P_{\sigma}(H)\right)$ is strongly transitive, there exists a block $C \in P_{\sigma}(H)$ such that $B \cup\{x\} \subset C$. Obviously $C \in \mathfrak{B}_{0}$ as $0 \in B \subset C$.

2. $\Rightarrow$ 1. Let $B_{1}=B\left(z_{1}, \ldots, z_{m}\right)$ and $B_{2}=B\left(x_{1}, \ldots, x_{n}\right)$ be two blocks of $P_{\sigma}(H)$ such that $B_{1} \cap B_{2} \neq \emptyset$ and moreover let $x \in B_{2}$. Our goal is to prove that there exists a block $C \in P_{\sigma}(H)$ such that $B_{1} \cup\{x\} \subset C$. We distinguish the two cases I) $0 \in B_{1} \cup B_{2}$ and II) $0 \notin B_{1} \cup B_{2}$.

I) By (22) we have $B_{1} \cup B_{2} \subset \gamma^{*}(0)$ because $0 \in B_{1} \cup B_{2}$ and $B_{1} \cap B_{2} \neq \emptyset$. Hence, in particular we have $B_{1} \in \mathfrak{B}_{0}$. Finally, by hypothesis, there exists a block $C \in \mathfrak{B}_{0} \subset P_{\sigma}(H)$ such that $B_{1} \cup\{x\} \subset C$.

II) We have $x \neq 0$ since $x \in B_{2}$ and $0 \notin B_{1} \cup B_{2}$. Moreover, if $b \in B_{1} \cap B_{2}$ then $b \neq 0$. Since $H$ is 0 -simple, $H x H=H=H b H$. Hence there exist two pairs $\left(y, y^{\prime}\right)$ and $\left(c, c^{\prime}\right)$ of elements of $H$ such that $z_{m} \in y x y^{\prime}$ and $x \in c b c^{\prime}$. Then, using the foregoing properties of the blocks in $P_{\sigma}(H)$ we obtain

$$
\begin{aligned}
x \in c b c^{\prime} & \subset c B\left(z_{1}, \ldots, z_{m}\right) c^{\prime} \\
& \subset B\left(c, z_{1}, \ldots, z_{m}, c^{\prime}\right) \\
& \subset B\left(c, z_{1}, \ldots, z_{m-1}, y, x, y^{\prime}, c^{\prime}\right) \\
& =B\left(z_{1}, \ldots, z_{m-1}, y, c, x, c^{\prime}, y^{\prime}\right) \subset B\left(z_{1}, \ldots, z_{m-1}, y, c, x_{1}, \ldots, x_{n}, c^{\prime}, y^{\prime}\right) .
\end{aligned}
$$

Moreover, we have

$$
\begin{aligned}
B\left(z_{1}, \ldots, z_{m}\right) & \subset B\left(z_{1}, \ldots, z_{m-1}, y, x, y^{\prime}\right) \\
& \subset B\left(z_{1}, \ldots, z_{m-1}, y, c, b, c^{\prime}, y^{\prime}\right) \subset B\left(z_{1}, \ldots, z_{m-1}, y, c, x_{1}, \ldots, x_{n}, c^{\prime}, y^{\prime}\right) .
\end{aligned}
$$

Hence $B\left(z_{1}, \ldots, z_{m}\right) \cup\{x\} \subset B\left(z_{1}, \ldots, z_{m-1}, y, c, x_{1}, \ldots, x_{n}, c^{\prime}, y^{\prime}\right)$ and the proof is complete.

From the previous theorem we obtain a sufficient condition for the transitivity of $\gamma$ in a 0 -simple semihypergroup:

Corollary 6.3 If $H$ is a 0-simple semihypergroup fulfilling one of the hypotheses in Theorem 6.2 then $\gamma$ is transitive.

Proof The claim follows immediately from Theorem 6.1 and the fact that $\gamma$ is the relation $\sim$ defined on the geometric space $\left(H, P_{\sigma}(H)\right)$.

We conclude this section by proving that the relation $\gamma$ is transitive in all simple semihypergroups. In order to attain that result, we make use of a special construction which is well known in the framework of semigroup theory, see e.g., [17]. If $H$ is a semihypergroup then we denote by $H_{0}$ the semihypergroup built by adding a zero scalar element $0 \notin H$ to $H$; the hyperproduct in $H_{0}$ extends naturally the one defined in $H$. We will refer to $H_{0}$ as the natural 0 -extension of $H$. The following propositions are immediate:

- $H$ is a simple semihypergroup if and only if $H_{0}$ is 0 -simple.

- Denote by $\beta_{H}$ and $\beta_{H_{0}}$ the fundamental relations $\beta$ associated respectively to $H$ and $H_{0}$. Then $\beta_{H_{0}}=$ $\beta_{H} \cup\{(0,0)\}$. In particular, $\beta_{H_{0}}$ is transitive if and only if $\beta_{H}$ is transitive.

- Denote by $\gamma_{H}$ and $\gamma_{H_{0}}$ the fundamental relations $\gamma$ associated respectively to $H$ and $H_{0}$. Then $\gamma_{H_{0}}=$ $\gamma_{H} \cup\{(0,0)\}$. In particular, $\gamma_{H_{0}}$ is transitive if and only if $\gamma_{H}$ is transitive.

With the help of the natural 0-extension of a semihypergroup, the proof of our last theorem becomes almost immediate; the reader could note that, in order to prove a relevant property of simple semihypergroups, we rely on a related property on 0 -simple semihypergroups. Actually, that procedure can help to extend the proof to different objects, and has been used also elsewhere, see e.g., [17,18], in related contexts.

Theorem 6.4 If $H$ is a simple semihypergroup then the relation $\gamma$ is transitive.

Proof Since $H$ is simple, then its natural 0-extension $H_{0}$ is 0 -simple. Moreover, $\gamma_{H_{0}}^{*}(0)=\{0\}$. As a consequence, $H_{0}$ fulfills the second condition of Theorem 6.2, whence $\gamma_{H_{0}}$ is transitive thanks to Corollary 6.3, and the claim follows.

As recalled by the first example in Sect. 2, the class of simple semihypergroups includes, as special subclasses, that of hypergroups and that of semihypergroups of type $U$ on the right. Transitivity of $\gamma$ in such subclasses has been shown in [11] and [13], respectively. Hence, the foregoing theorem provides an extension of the above mentioned results. Moreover, it is worth noting that all results in this section obviously hold also for simple and 0-simple semigroups, as defined e.g., in [17]. 


\section{Conclusions and open problems}

The class of semihypergroups is huge, even if one considers semihypergroups having rather small order. Within this class there are instances having rather unexpected properties, and certain implications that hold true e.g., for semigroups or hypergroups do not hold on semihypergroups. We believe that the manifold examples shown in this paper may improve knowledge of semihypergroups and help further investigations in this subject.

For example, the 34 semihypergroups described in Remark 4.3, see (17), (18) and (19), and their respective transposed semihypergroups, are strongly simple semihypergroups having a subsemihypergroup $K=$ $\{a, b, c\}$ where $\beta$ is not transitive. Moreover, they also have two subsemihypergroups, $K_{1}=\{a, b, d\}$ and $K_{2}=\{a, c, d\}$, where $\beta$ is transitive. This fact opens the problem to prove whether or not there exists a strongly simple semihypergroup all whose subsemihypergroups $K$ with $|K| \geq 3$ have a relation $\beta$ which is not transitive.

Acknowledgments D. Fasino was supported by PRIN project no. 20083KLJEZ "Problemi di algebra lineare numerica strutturata: analisi, algoritmi e applicazioni”. D. Freni was supported by PRIN project no. 20087P8MN2 "Teoria dei disegni, teoria spettrale dei grafi, teorie combinatorie e loro applicazioni”.

Open Access This article is distributed under the terms of the Creative Commons Attribution License which permits any use, distribution, and reproduction in any medium, provided the original author(s) and the source are credited.

\section{References}

1. Anvariyeh, S.M.; Davvaz, B.: Strongly transitive geometric spaces associated to hypermodules. J. Algebra 322, 1340-1359 (2009)

2. Chaopraknoi, S.; Triphop, N.: Regularity of semihypergroups of infinite matrices. Thai J. Math. 4, 7-11 (2006)

3. Davvaz, B.: Characterizations of sub-semihypergroups by various triangular norms. Czechoslovak Math. J. 55, 923$932(2005)$

4. Davvaz, B.: Applications of the $\gamma^{*}$-relation to polygroups. Commun. Algebra 35, 2698-2706 (2007)

5. De Salvo, M.: $K_{H}$-hypergroups. Atti Sem. Mat. Fis. Univ. Modena 31, 112-122 (1982, in Italian)

6. De Salvo, M.; Freni, D.: Cyclic semihypergroups and hypergroups. Atti Sem. Mat. Fis. Univ. Modena 30, 44-59 (1981, in Italian)

7. De Salvo, M.; Fasino, D.; Freni, D.; Lo Faro, G.: Isomorphism classes of the hypergroups of type $U$ on the right of size five. Comp. Math. Appl. 58, 390-402 (2009)

8. Fasino, D.; Freni, D.: Existence of proper semihypergroups of type $U$ on the right. Discrete Math. 307, 2826-2836 (2007)

9. Fasino, D.; Freni, D.: Minimal order semihypergroups of type $U$ on the right. Mediterr. J. Math. 5, 295-314 (2008)

10. Freni, D.: $r$-Hypergroups and their extensions. Atti Società Peloritana Sci. Fis. Mat. Natur. 27, 77-93 (1981)

11. Freni, D.: A new characterization of the derived hypergroup via strongly regular equivalences. Commun. Algebra 30(8), 3977-3989 (2002)

12. Freni, D.: Strongly transitive geometric spaces: applications to hypergroups and semigroups theory. Commun. Algebra 32(3), 969-988 (2004)

13. Freni, D.: Minimal order semihypergroups of type $U$ on the right, II. J. Algebra 340, 77-89 (2011)

14. Gutan, C.: Plongements d'un hypergroupe dans un hypergroupe ayant un élément de plus. Riv. Mat. Pura Appl. 18, 8592 (1996)

15. Gutan, C.: Extensions des semigroupes associées à certains semihypergroupes très fins. In: Algebraic Hyperstructures and Applications (Prague, 1996), 43-52. Democritus Univ. Thrace, Alexandroupolis (1997)

16. Gutan, M.: On the transitivity of the relation $\beta$ in semihypergroups. Rend. Circ. Mat. Palermo (2) 45, 189-200 (1996)

17. Howie, M.: Fundamentals of Semigroup Theory. Oxford University Press, New York (1995)

18. Jafarabadi, H.M.; Sarmin, N.H.; Molaei, M.R.: Completely simple and regular semi hypergroups. Bull. Malaysian Math. Sci. Soc. 35, 335-343 (2012)

19. Koskas, H.: Groupoïdes, demi-hypergroupes et hypergroupes. J. Math. Pures Appl. 49, 155-192 (1970)

20. Vougiouklis, T.: The set of hypergroups with operators which are constructed from a set with two elements. Acta Univ. Carolin. Math. Phys. 22, 7-10 (1981)

21. Vougiouklis, T.: Fundamental relations in hyperstructures. Bull. Greek Math. Soc. 42, 113-118 (1999) 